Word count: 7402

Dr. William Mandy ${ }^{1}$

Ms. Adele Wang ${ }^{2}$

Dr. Irene Lee ${ }^{2}$

Prof. David Skuse ${ }^{2,3}$

${ }^{1}$ Research Department of Clinical, Educational and Health Psychology, UCL, London, UK

${ }^{2}$ Behavioural and Brain Science Unit, UCL, Institute of Child Health, London, UK

${ }^{3}$ Department of Child and Adolescent Mental Health, Great Ormond Street Hospital, London, UK

Running head: Evaluating social (pragmatic) communication disorder

Conflict of Interest: Professor Skuse is a stockholder in IxDx, the company that owns the 3Di. 


\begin{abstract}
Background: Social (pragmatic) communication disorder (SPCD) is a new diagnosis introduced by DSM-5, characterised by problems with verbal and non-verbal social communication. It is currently unclear whether SPCD is a valid diagnostic category, because little is known about the characteristics of those who meet its criteria. We sought to identify and describe cases of SPCD, to contribute to debates about its validity. We investigated whether the symptoms of SPCD cluster together to form a coherent syndrome that is distinct from autism spectrum disorder (ASD) in terms of its core and associated features.
\end{abstract}

Methods: Participants were young people $(\mathrm{N}=1081$, age range $=4-18$ years $)$ who had attended a specialist social communication disorders clinic for children with fluent language and normal-range intelligence. Standardised parent-report data were collected using the Developmental, Dimensional and Diagnostic Interview (3Di), Child Communication Checklist (CCC) and Strengths and Difficulties Questionnaire (SDQ). An algorithm was designed using 3Di and CCC items to implement DSM-5 SPCD criteria.

Results: Eighty-eight young people met our criteria for SPCD, with 801 meeting DSM-5 ASD criteria and the remaining 192 having neither SPCD nor ASD ('clinical comparison group'). The core symptoms of SPCD co-occurred to a moderate degree (average inter-item correlation=.22). SPCD cases had autistic social difficulties that were intermediate between ASD and the clinical comparison group. SPCD was associated with high rates of non-autistic psychopathology, with $63.5 \%$ scoring in the abnormal range of the SDQ's Total Problems scale.

Conclusions: We did not find evidence that SPCD is qualitatively distinct from ASD. Rather, it appears to lie on the borderlands of the autism spectrum, describing those with autistic traits that fall just below the threshold for an ASD diagnosis. SPCD may have clinical utility 
for identifying people with autistic traits that are insufficiently severe for ASD diagnosis, but who nevertheless require support.

Keywords: Social (pragmatic) communication disorder (SPCD); autism spectrum disorder (ASD); Diagnostic and Statistical Manual, Fifth Edition (DSM-5); pervasive developmental disorder - not otherwise specified (PDD-NOS); nosology

Abbreviations: ASD (autism spectrum disorder); CCC (Child Communication Checklist); SDQ (Strengths and Difficulties Questionnaire); PDD (pervasive developmental disorder); PDD-NOS (pervasive developmental disorder - not otherwise specified); SPCD (social (pragmatic) communication disorder) 


\section{EVALUATING SOCIAL (PRAGMATIC) COMMUNICATION DISORDER}

The Fifth Edition of the Diagnostic and Statistical Manual (DSM-5) posited the existence of a hitherto unrecognised neurodevelopmental disorder, called social (pragmatic) communication disorder (SPCD) (American Psychiatric Association [APA], 2013). The essential feature of this new clinical entity is a persistent difficulty with the social use of verbal and nonverbal communication, manifesting as (1) problems using communication for social purposes, (2) impaired ability to adapt communication to context, (3) difficulty following conversational and narrative conventions and (4) difficulty inferring what is not explicitly stated. For a diagnosis of SPCD to be made, these symptoms must limit social, academic, or occupational functioning; must originate early in development; and cannot be attributable to other difficulties such as autism spectrum disorder (ASD), intellectual disability or clinically impaired word structure and grammar (APA, 2013).

Because SPCD was only recently added to psychiatric nosology, little is known about its core and associated characteristics. In consequence, there is minimal evidence by which to judge whether SPCD is a valid diagnosis, leading some to ask whether its introduction to DSM-5 was precipitous (e.g., Bruckner-Wertman, Laor \& Golan, 2016; Norbury, 2014). In the current study, we seek to investigate the characteristics of young people meeting preliminary criteria for SPCD, in order to contribute to debates about the validity of this new and untested DSM-5 diagnostic construct.

A mental disorder diagnosis is a working hypothesis that a set of signs and symptoms cluster together as a syndrome because they are all manifestations of a specific underlying disease process (Rutter, 1978). Thus, it is a necessary (but not sufficient) criterion for validity that the signs and symptoms of a proposed diagnosis should statistically cluster together (Robins \& Guze, 1979). We know of no published findings on the coherence of SPCD as a syndrome. 
Thus it is the first aim of the current study to investigate the extent to which the diagnostic features of SPCD hang together, statistically.

In addition to the requirement of syndromal coherence, to be considered valid, a diagnostic category must be distinct from other disorders and from typical development in terms of its core (diagnostic) features and its associated characteristics (Kendler, 1980). The question of whether SPCD can be meaningfully and consistently distinguished from other disorders and from typical development remains to be answered (e.g., Lord \& Bishop, 2015). In particular, a crucial consideration is how SPCD relates to, and can be differentiated from, ASD (Bruckner-Wertman et al., 2016). It is the second aim of the current study to investigate how young people with SPCD differ from those with ASD and from a clinical comparison group (without ASD or SPCD) in terms of ASD symptoms and associated difficulties.

When considering how SPCD is distinct from ASD, several commentators have proposed that SPCD may represent a specific pattern of symptoms comprising autistic social communication problems in the absence of repetitive behaviours (e.g., Happé, 2011; Lord \& Bishop, 2015). Before DSM-5, this symptom profile was sufficient for an autism spectrum diagnosis, captured by the DSM-IV-TR ASD subtype 'pervasive developmental disorder not otherwise specified' (PDD-NOS) (APA, 2000). In fact, the great majority of PDD-NOS cases showed this specific symptom profile (Mandy, Charman, Gilmour \& Skuse, 2011). Unlike DSM-IV-TR, DSM-5 stipulates that for a diagnosis on the autism spectrum both social communication impairment and repetitive behaviour be present; and so excludes a substantial proportion with a PDD-NOS symptom profile (Huerta, Bishop, Duncan \& Lord, 2012). It has been suggested that SPCD was specifically designed to encompass individuals who met criteria for PDD-NOS in DSM-IV-TR, but who are excluded from the autism spectrum under modified DSM-5 ASD criteria, due to a lack of repetitive behaviour (Happé, 2011; Lord \& Bishop, 2015; Swineford, Thurm, Baird, Wetherby \&Swedo, 2014). 
The only published empirical study so far to investigate directly DSM-5 SPCD offers support for the idea that it encompasses the specific profile of autistic symptoms (i.e., social communication problems without repetitive behaviours) that was previously captured by PDD-NOS (Kim et al., 2014). In this South Korean epidemiological study, around a third of DSM-IV-TR PDD-NOS cases (17/58, 29\%) did not qualify for an ASD diagnosis when DSM-5 rules were applied; and most of these (13/17, 76\%) met criteria for SPCD. Nevertheless, it is notable that the majority of SPCD cases identified by Kim and colleagues had not previously had a DSM-IV-TR autistic diagnosis. This raises the possibility that SPCD could apply well beyond PDD-NOS to include diverse individuals who are experiencing social difficulties. As such it may represent a rather heterogeneous group, who are not, as a whole, distinguished by any one pattern of autistic symptoms (Norbury, 2014). To increase understanding of SPCD, and whether it represents a specific profile of autistic symptoms, it will be valuable to investigate the proportion of SPCD cases who previously had PDD-NOS, and the overall profile of social communication and repetitive behaviour features amongst those meeting criteria for SPCD.

In summary, SPCD is a new DSM-5 disorder about which little is known, and this prevents a sound estimation of its validity. Specifically, it is currently unclear whether SPCD is a coherent symptom cluster; and how it is distinguished from ASD. Therefore we aimed to provide a description of young people meeting preliminary SPCD criteria in order to contribute to debates about the nature and validity of this diagnostic entity. We designed an algorithm to implement retrospectively DSM-5 SPCD criteria based on standardised parent reports in a large sample of young people who had undergone clinical assessment for ASD. Individuals meeting these SPCD criteria were compared to young people meeting criteria for DSM-5 ASD and to those not meeting criteria for ASD or SPCD ('clinical comparison group'). We addressed the following questions: 
(1) To what extent do the core symptoms of SPCD cluster together?

(2) What is the overlap between PDD-NOS and SCPD?

(3) Can SPCD be distinguished from ASD and from a clinical comparison group on measures of autistic traits, internalising and externalising difficulties, and social impairment?

\section{METHODS}

\section{Participants}

Participants were 1081 consecutive referrals, aged between 4 and 18 years, receiving a parent-report autism assessment interview between April 1999 and August 2013 at a NHS clinic in England. The clinic is a specialist service for the assessment of young people with social communication problems. Referral criteria stipulate that clinic attendees should be in mainstream education and not have an intellectual disability. In addition, referrals are required to have fluent spoken language. Interviews were conducted by an experienced child and adolescent psychiatrist or clinical psychologist. This retrospective chart review study used non-identifiable data collected in the course of routine clinical assessment. Ethical permission was provided after review by the research and development office of the hospital in which this study was based, and appropriate measures were taken to ensure young person and parent confidentiality.

\section{Measures}

The Developmental, Dimensional and Diagnostic Interview (3Di) - All participants were assessed using the 3Di, a computerised, parent-report, semi-structured interview for the assessment of ASD and autistic traits. The 3Di has a diagnostic algorithm which draws on 122 items to implement DSM-IV-TR / ICD-10 diagnostic criteria for pervasive 
developmental disorders. This algorithm has excellent reliability, demonstrated by high levels of inter-rater and test-retest agreement (intraclass correlation coefficients $>.86$ ) (Skuse et al., 2004). The 3Di also has strong criterion validity with respect to both clinician diagnosis and the Autism Diagnostic Interview - Revised (ADI-R) (Skuse et al., 2004). It measures the range of symptoms relevant to DSM-5 diagnostic criteria for ASD (Mandy, Charman \& Skuse, 2012).

The 122 items used in the 3Di's DSM/ICD algorithm are a subset of a larger pool $(n>200)$ of ASD-relevant items in the 3Di, which cover a broad range of autistic features including sensory abnormalities, pragmatic language, and associated motor and behavioural features of ASD. The SPCD and DSM-5 ASD algorithms described below drew from this wider collection of items (including those in the DSM/ICD algorithm), to allow for a comprehensive implementation of DSM-5 diagnostic criteria.

The Child Communication Checklist (CCC) - The first edition of the CCC (Bishop, 1998) was administered to all participants, and its items contributed to the SPCD and ASD algorithms described below. The CCC is a widely-used and well validated measure of the pragmatic aspects of young people's communication difficulties (Bishop, 1998; Bishop \& Baird, 2001). It comprises five subscales assessing different aspects of pragmatic difficulties, which can be summed into an overall 'pragmatic composite' score. In addition the CCC has subscales that measure intelligibility of speech ('Speech'), complexity of spoken grammar ('Syntax'), autistic social problems (Social Relationships') and restricted interests ('Interests').

The Strengths and Difficulties Questionnaire (SDQ) - The SDQ is made up of 25 items, arranged into five subscales measuring conduct problems, hyperactivity (i.e., attention deficit hyperactivity disorder symptoms), emotional problems, peer problems and prosocial 
behaviour (Goodman, 1997). It also has a total problems score, which is the sum of all subscales except the prosocial subscale. In the current study, the parent-report version was used. The SDQ has established reliability and validity as a screening instrument for mental disorders in childhood (Goodman, 2001). The parent-report version of the SDQ is a valid measure of psychopathology in ASD populations (e.g., Simonoff et al., 2013).

\section{Procedure}

Individuals were classified using algorithms whereby 3Di and CCC items were mapped onto specific diagnostic criteria, and summed according to pre-specified rules that reflect DSM-5 rubric. In DSM-5, SPCD criterion A ('social use of verbal and nonverbal communication') has four sub-criteria: (A1) Deficits in using communication for social purposes; (A2) Impairment of ability to change communication to match context or the needs of the listener; (A3) Difficulties following rules for conversation and storytelling; and (A4) Difficulties understanding what is not explicitly stated. For each of these, DSM-5 gives examples of the specific behaviours and difficulties that would indicate that a sub-criterion is met. A panel of three of the authors (WM, DS, AW) selected items from the 3Di and CCC that mapped onto each SPCD sub-criterion, based on the examples given in DSM-5. Six items were selected for each sub-criterion, so that a total of 24 items were mapped onto SPCD criterion A. This SPCD algorithm is shown in Table 1. The majority (19) of criterion A items were from the CCC scales measuring pragmatic competence, with the remainder being original 3Di items. For any individual to meet criterion A of SPCD they had to have at least one definite example of a difficulty from each of the four sub-criteria. A further six items (two from the CCC, four from the $3 \mathrm{Di}$ ) were used to assess criterion $\mathrm{B}$, which states that functional impairment must be present in the social, educational and/or occupational domain. For criterion $\mathrm{B}$ to be met in our algorithm, at least one item concerning functional impairment had to be endorsed as 'definitely'. 
One key exclusion criterion for SPCD is that the individual does not have DSM-5 ASD. There is imperfect overlap between DSM-5 ASD and DSM-IV-TR PDD, so we used the 3Di's DSM-5 ASD algorithm to implement this exclusion criterion. This is hierarchical, and draws upon 52 items from the $3 \mathrm{Di}(\mathrm{N}=31)$ and $\mathrm{CCC}(\mathrm{N}=21)$ which map onto the DSM-5 description of ASD. Criterion A (impaired 'social communication and social interaction') of ASD has three sub-criteria concerning (1) social-emotional reciprocity; (2) non-verbal communication in social interaction; and (3) relationships. Each of these is described in DSM-5 with several examples of how it could be met. Based on these examples we broke each sub-criterion down into sub-several components and then mapped three 3Di/CCC items onto each component. For example the components of the relationships sub-criterion were 'adjusting to social contexts', 'shared play and imagination' and 'friendship and social interest', each of which was assessed by a cluster of three 3Di/CCC items. For a sub-criterion to be met, at least one of its components had to be fulfilled. A component was deemed to be fulfilled if all three of its 3Di/CCC items were endorsed; or if at least two of its items were endorsed, with at least one of these being 'definite' (as opposed to 'possible'). Criterion B of ASD ('restricted, repetitive patterns of behaviour, interests, or activities') has four subcriteria in DSM-5: (1) Stereotyped behaviour or speech; (2) insistence on sameness; (3) restricted, fixated interests; (4) hyper- or hyporeactivity to sensory input. These were assessed by the in 3Di ASD algorithm in a similar hierarchical fashion as was used for criterion A: each of B's sub-criteria were split into components based on the text of DSM-5, and 3Di/CCC items were mapped onto each of these. Each B sub-criterion was split into two components, and each was assessed using two 3Di/CCC items. A component was deemed fulfilled if both of its items were endorsed; or if one was endorsed as 'definite'. In line with DSM-5, ASD was diagnosed if a person fulfilled all three social communication and social interaction sub-criteria, and at least two restricted behaviour and speech sub-criteria. 
In this study, we were interested to compare DSM-5 and DSM-IV-TR diagnostic outcomes. Therefore individuals were also described using the 3Di's DSM-IV-TR PDD algorithm, as described in detail in other studies (Mandy et al., 2011; 2012). Individuals who showed the full triad of impairment in addition to delayed onset of first words or phrase speech were classified as having autistic disorder. People with the triad of impairments who did not have reported delay in language development were classified as Asperger's disorder (Szatmari, 2000). PDD-NOS was assigned to people who did not meet criteria for autistic disorder of Asperger's, who scored in the 3Di clinical range for social impairment, and for either communication impairment or repetitive, stereotyped behaviour.

Analysis

To investigate the extent to which the core symptoms of SPCD cluster together we examined the internal consistency of our proposed SPCD algorithm. To this end we calculated the average inter-item correlation, controlling for age, for the 24 items measuring SPCD core symptoms (i.e., criterion A). As described above (in the 'Procedure' subsection), SPCD criterion A has four diagnostic sub-criteria, each of which was measured by six items, summed to create algorithm subscales. We calculated average inter-item correlations, controlling for age, for each of these subscales, and for the six items used to map criterion B (i.e., functional impairment). This method of assessing internal consistency using average inter-item correlations is superior to Cronbach's $\alpha$ as, unlike Cronbach's $\alpha$, it is not directly influenced by the number of items in a scale (Tavakol \& Dennick, 2011). Also, it allowed us to control for any confounding effects of age. By convention, average inter-item correlations in the range of .15-.50 indicate acceptable internal consistency (Clark \& Watson, 1995).

To estimate how well individual items map onto SPCD criteria, we calculated how strongly each item correlated with: (1) the total SPCD criterion A algorithm score, and (2) the 
summed score of its subscale, controlling for age. Agreement between DSM-IV-TR and DSM-5 diagnostic outcomes was tested using Kappa. Group differences on continuous variables were tested using one-way ANOVAs, with any significant main effects investigated using post-hoc Scheffe tests. Group comparisons on categorical variables were made by calculating odds ratios with $95 \%$ confidence intervals. All analyses were conducted using STATA 14 (StataCorp, 2015).

\section{RESULTS}

Question 1: Do the symptoms of SPCD cluster together?

For the 24-items mapping onto SPCD criterion A, the average inter-item correlation (controlling for age) was .22, which is in the acceptable range, albeit at its lower end. For the four sub-criteria of the SPCD algorithm, average inter-item, age-controlled correlations were as follows: (A1) deficits in using communication for social purposes $=.17$; (A2) difficulty changing communication to match context $=.21$; (A3) difficulties following rules for conversation and storytelling $=.21$; and (A4) difficulties understanding what is not explicitly stated $=.27$. For the six items measuring functional impairment SPCD criterion B), the average inter-item, age-controlled correlation was .15.

\section{[Table 1]}

Table 1 shows item-total correlations for the 24 items used to measure SPCD criterion A symptoms, controlling for age. It also presents item-subscale, age-controlled correlations for the four criterion A subscales and the six items measuring SPCD criterion B. Most (24/30, $80 \%$ ) item-total correlations, and all item-subscale correlations, were moderate or above ( $r>4)$. Partial correlations between the SPCD criterion A subscales, controlling for age, were all highly significant $(\mathrm{p}<.0001)$ and ranged from $\mathrm{r}=.43$ to $\mathrm{r}=.52$. 
Question 2: What is the overlap between PDD-NOS and SPCD?

Table 2 shows the overlap between the original 3Di algorithm which implements DSM-IVTR criteria, and the DSM-5 algorithms designed to classify SPCD and ASD. Before considering the specific question of the overlap between PDD-NOS and SPCD, it is interesting to note the degree of concordance between DSM-IV-TR and DSM-5 ASD diagnosis. There was moderate agreement $(83.2 \%$ concordance, Kappa $=.55)$ between DSMIV-TR and DSM-5 algorithms for identifying ASD. Overall sensitivity of DSM-5 ASD judged against the criterion of DSM-IV-TR PDD diagnosis was .88; and specificity was .68. Sensitivity for DSM-IV-TR autistic disorder (.94) and Asperger's (.94) disorder was high. For PDD-NOS it was .71.

\section{[Table 2]}

As is shown in Table 2, a minority of SPCD cases $(n=26 / 88,29.5 \%)$ were participants who met criteria for DSM-IV-TR PDD-NOS, but not DSM-5 ASD. All of these had received a PDD-NOS diagnosis because they had clinically severe social and communication problems, but did not score above the clinical threshold for repetitive and stereotyped behaviour. A further 24 SPCD cases were individuals who had received a specified PDD diagnosis (autism or Asperger's disorder) by DSM-IV-TR rubric, but did not meet criteria for DSM-5 ASD. They had all narrowly missed meeting DSM-5 ASD criteria, either because they met two, rather than all three 'social communication' criteria ( $n=14 / 24,58.3 \%)$; or because they met one, rather than two, repetitive stereotype behaviour criteria $(10 / 24,41.7 \%)$.

The remainder of the SPCD $(38 / 88,43.2 \%)$ cases had not met DSM-IV-TR criteria for any PDD. These were mostly young people who had narrowly missed meeting criteria for ASD either because they had sufficient social impairments but a lack of repetitive stereotype 
behaviours $(11 / 38,29.0 \%)$; or because they had repetitive behaviours without meeting all three social criteria $(18 / 38,47.4 \%)$.

We examined the SPCD algorithm scores of the 192 clinical comparison participants, i.e., those who did not meet criteria for SPCD or ASD. Of these, 76 had narrowly missed meeting SPCD criteria. In most of these cases $(n=75)$, the young person showed functional impairment (i.e., met SPCD criterion B), but only met three of the four sub-criteria of SPCD criterion A. In one case, the young person met all sub-criteria of criterion A, but did not have any reported functional impairment as stipulated for criterion B. Four clinical comparison participants had three criterion A symptoms in the absence of functional impairment. The rest either had two $(n=54)$, one $(n=33)$ or no $(n=25)$ SPCD criterion A symptoms.

In DSM-5, ASD precludes an SPCD diagnosis. Nevertheless we were interested to see the SPCD symptom counts for participants with ASD. All ASD cases showed sufficient functional impairment to meet SPCD criterion B. Three quarters (600/801) also met SPCD criterion A, showing all four symptom types. There were a further 146 ASD participants who had three of the four SPCD criterion A symptoms. The remaining ASD cases met two $(n=40)$, one $(n=13)$ or no $(n=2)$ SPCD criterion A symptoms.

Question 3: Can SPCD be distinguished from ASD and from a clinical comparison group on clinically relevant variables?

Table 3 shows a comparison of groups on demographic characteristics and measures of intelligibility and complexity of expressive language (CCC Speech and Syntax) and autistic traits (CCC Social Relationships, CCC Interests). Because multiple CCC pragmatics items were used in the SPCD algorithm, we do not report data on the CCC pragmatics scales, to avoid circularity. Instead we report measurement scales independent to those used to assign SPCD diagnosis. 
SPCD was similar to ASD and non-ASD in terms of age and gender ratio. In terms of CCC Speech and Syntax, the SPCD group showed similar scores to the ASD group; and lower scores (denoting greater impairment) than the non-ASD group. On the CCC measure of autistic social difficulties (Social Relationships) the SPCD group mean was intermediate between ASD and non-ASD. A somewhat different pattern was observed for the CCC Interests scale, which assesses autistic restricted interests. On this scale the SPCD group scored lower than ASD, but similarly to the non-ASD group. This contrasting pattern of group differences for autistic social and non-social traits is shown in Figure 1, in which the Social Relationships and Interests scores are standardised as z-scores for ease of comparison.

\section{[Figure 1]}

To see whether SPCD could be distinguished from ASD and from non-ASD clinical controls by its associated pattern of psychopathology and adaption, we examined SDQ scores. Due to changes in clinic practice over time, SDQ scores were only available for a subsample $(n=612 / 1081,56.6 \%)$. There was no association between whether SDQ scores were available and DSM-5 diagnosis, gender, age or symptom severity on the DSM-5 algorithm (all ps>.269).

[Table 4]

As is shown in Table 4, we compared the DSM-5 groups according to the proportion of participants scoring in the clinical range on the SDQ Total Problems scale and each SDQ subscale. Generally, SPCD was intermediate between ASD and non-ASD in terms of the proportion of participants who showed significant difficulties on the SDQ.

\section{DISCUSSION}


We sought to describe young people meeting preliminary criteria for SPCD, based on systematic parent reports, in order to contribute to debates about the validity of this new DSM-5 diagnostic category. In particular we were interested to discover whether DSM-5 diagnostic criteria for SPCD describe a syndrome (i.e., a set of symptoms that cluster together), and to understand better how young people with SPCD differ from those with ASD.

We found preliminary evidence that SPCD, as measured by our algorithm, comprises symptoms that statistically cluster together. Average inter-item correlations for the total scale $(\mathrm{r}=.22)$ and for each subscale $(\mathrm{rs}=.17-.27)$ were at a level that is conventionally considered indicative of adequate internal consistency (e.g., Clark \& Watson, 1995). Item-total and itemsubscale correlations were all significant, and were mostly moderate ( $r>.4)$ or above. Also, correlations between the four algorithm subscales measuring each diagnostic sub-criterion for SPCD were in the moderate to large range ( $\mathrm{rs}=.43-.52)$, controlling for age. This degree of association amongst sub-dimensions of SPCD compares favourably with that of ASD (e.g., Happé, Ronald \& Plomin, 2006).

This is, to our knowledge, the first study to present data on the statistical associations between SPCD symptoms. For this reason further work will be required before it can be concluded that SPCD symptoms cluster together to form a coherent syndrome. It will be especially helpful to triangulate our findings against those based on other samples and algorithms. The DSM-5 description of SPCD divides its core (criterion A) symptoms into four types, thus suggesting a four-factor structure. Studies that combine exploratory and confirmatory factor analysis to investigate the structure of SPCD are required to test this. Such work would extend the current research by providing more information on how closely, and in which configuration, the symptoms of SPCD hang together. It could also elucidate how SPCD characteristics map onto the social and communication domain of the ASD dyad. 
One potential limitation of SPCD is that it may be a broad, non-specific category that is applicable to diverse individuals who experience social problems but who do not have ASD. Our findings contradict this notion. SPCD, as diagnosed by our algorithm, was actually quite rare in the current sample, with only 88 young people ( $8.1 \%$ of total sample) meeting criteria, whilst a larger group ( $\mathrm{n}=192,17.8 \%$ of total sample) received neither a SPCD nor an ASD diagnosis. This is notable because all participants had attended a clinic due to concerns about their social communication: therefore SPCD appear to describe only a minority of young people presenting clinically with social problems outside of ASD. This is consistent with the only other empirical study to have implemented DSM-5 SPCD criteria, which found that, of 108 children identified as having social problems but who did not meet ASD criteria, only 20 (18.5\%) had SPCD (Kim et al., 2014).

A key uncertainty about SPCD is how it relates to ASD (Lord \& Bishop, 2015). One possibility is that it represents 'mild autism', offering a diagnostic home to those with elevated autistic social and non-social (i.e., repetitive behaviour) traits, but who do not meet ASD criteria because their symptoms are not quite severe enough (Bruckner-Wertman et al., 2016). Another possibility is that SPCD applies to those with a specific symptom profile of severe autistic social and communication problems, in the absence of repetitive and stereotyped behaviours (hereafter we call this profile 'SC+RSB-') (Swineford et al., 2016). These individuals would have previously qualified for PDD-NOS, but were excluded from the autism spectrum by DSM-5's insistence that both social and non-social symptoms be present for an ASD diagnosis (Happé, 2011).

We found that SPCD does encompass individuals with the SC+RSB- profile. This included 26 young people $(29.5 \%$ of SPCD cases) who met PDD-NOS criteria under DSM-IV-TR rules, as well as others ( $\mathrm{n}=22,25.0 \%)$ who narrowly missed out on an ASD diagnosis because they met criteria for social impairments but had insufficient repetitive and 
stereotyped behaviours. This was reflected in the fact that the SPCD group was differentiated from ASD, but not from the clinical comparison group, by scores on the CCC's Interests subscale, which assesses autistic focused and inflexible interests. Nevertheless, around a third of SPCD cases $(32 / 88,36.4 \%)$ actually had sufficient repetitive stereotyped behaviours for an ASD diagnosis, but narrowly fell short on the social and communication domain. We consider such people to have 'mild autism', since they have a range of autistic social and non-social difficulties that are of insufficient severity for a full ASD diagnosis. Thus we found no single pattern of symptoms that characterised SPCD. Rather, it appears to be a diagnosis for various people with 'nearly autism', on the borderlands of the autism spectrum (Bishop \& Norbury, 2002).

The fact that SPCD encompasses various 'nearly autism' symptom profiles makes it useful for meeting two challenges to categorising autistic difficulties. First, because SPCD incorporates the $\mathrm{SC}+\mathrm{RSB}$ - profile, it helps accommodate the fact that autistic social and nonsocial symptoms are not strongly associated, such that some children have one type of autistic difficulty without showing the full syndrome (Happe et al., 2006; Mandy \& Skuse, 2008). Second, ASD is a dimensional disorder, yet diagnosis is a categorical decision, making it inevitable that some people with elevated autistic difficulties will be denied an ASD diagnosis (Constantino \& Todd, 2003). SPCD provides a diagnostic home for these 'mild autism', sub-threshold cases.

Conceptually, SPCD has much in common with Pragmatic Language Impairment (PLI), a putative syndrome characterised by a primary deficit in the use of language in social context (Bishop, 1998; Baird \& Norbury, 2015). Gibson, Adams, Lockton \& Green (2013) compared children with ASD, PLI and specific language impairment (SLI), finding that those with PLI were distinguished from SLI and ASD by a distinct profile of difficulties; namely elevated peer interaction problems and low levels of repetitive and stereotyped behaviour. This PLI 
symptom profile matches the $\mathrm{SC}+\mathrm{RSB}$ - profile we describe above, lending credence to the notion that SPCD will apply to many with PLI. The current study was based in a clinic that does not conduct standardised language assessments with all referrals, and so we are unable to investigate the overlap between SPCD and PLI. In future, in-person assessments, that incorporate direct and standardised observations of structural and pragmatic language will be required to understand the relationship between PLI and SPCD (Swineford et al., 2014). This work should address the extent to which SPCD occurs in the absence of formal language difficulties; and whether pragmatic difficulties seen in SPCD are actually part of a broader array of social and communication problems that map onto the 'social communication and social interaction' construct of DSM-5 ASD (Baird \& Norbury, 2016).

Investigation of associated psychopathology, as measured by parent report on the SDQ, further supports the idea that SPCD is a condition on the borderlands of the autism spectrum, midway between ASD and non-ASD. There was no evidence for a SPCD-specific pattern of associated psychopathology. Rather, for overall level of psychopathology, and on focused measures of emotional problems (i.e., anxiety and depression), conduct problems, and social impairments, SPCD scored intermediate between ASD and the clinical comparison group. It was notable that the participants with SPCD showed very high levels of psychopathology, with two-thirds scoring in the abnormal range for the SDQ's 'Total Problems' scale, where as in the general population only $10 \%$ of children score at this level. This suggests that those meeting criteria for SPCD show high levels of clinical need, that will need to be acknowledged by clinical services, insurance companies and education authorities (Lord \& Bishop, 2015).

\section{Limitations}


Notwithstanding the strengths of the current study, which include a large sample size, the use of standardised data and the comparison of groups on measures independent of those used to assign group status, our findings must be viewed in the light of the following limitations. First, in this retrospective chart review, we only used parent-report data: our methods of case identification do not meet the gold-standard for the assessment of autism and related conditions (NICE, 2011). Future investigations will require direct observation and testing, to confirm and extend our findings (Swineford et al., 2015). This will be important for assigning ASD and SPCD diagnosis, and for evaluating key variables (e.g., autistic symptoms, language, IQ) than may differentiate SPCD from ASD, PLI and from other developmental outcomes. A second, related point is that we did not have direct assessments of IQ and language development, and so it is possible that some young people who we identified as having SPCD actually had pragmatic difficulties arising from an intellectual disability or specific language impairment (SLI). The clinic from which participants came does not accept referrals of those with intellectual disability and/or SLI, but it is possible that some such unidentified cases could have been included. Third, our focus on a sample with fluent language and without intellectual disability limits the generalisabilty of our findings across the whole autism spectrum. Fourth, we used a heterogeneous clinical comparison group. In future studies, more focused comparisons should be made, to understand whether SPCD is clearly differentiated from the social difficulties seen in other types of psychopathology, such as attention deficit/hyperactivity disorder (St Pourcain et al., 2012), conduct problems (Mandy, Skuse, Steer, St Pourcain \& Oliver, 2013) and social anxiety (Pickard, Rijsdijk, Happé \& Mandy, 2017).

\section{Conclusions}

Notwithstanding the above limitations, the current study has extended knowledge about SPCD, a new and untested DSM-5 diagnostic entity. Our findings do not support the idea that 
SPCD is a qualitatively distinct entity from ASD. Rather, it appears to exist on the border between ASD and non-ASD, providing a diagnostic home for people who have marginally sub-threshold autistic traits. Young people meeting criteria for SPCD in this clinic sample showed high levels of diverse (internalising and externalising) psychopathology and social impairment, suggesting that they have substantial needs that should be taken seriously by clinical and educational services. For this reason, SPCD may possess clinical utility as a way of identifying people who have autistic difficulties that are impairing, but insufficiently severe for an ASD diagnosis.

\section{ACKNOWLEDGEMENTS}

We would like to thank the young people and parents who participated, and all the clinicians and administrators who conducted and facilitated the assessments on which the current study is based.

\section{Correspondence to:}

Dr Will Mandy, Department of Clinical Psychology, UCL, Gower Street, London, WC1E 6BT, UK. Telephone: 44 (0)207 679 1897. Fax: 44 (0)207 916 1989.w.mandy@,ucl.ac.uk 


\section{REFERENCES}

American Psychiatric Association (2000). The Diagnostic and Statistical Manual, Fourth Edition Text Revision (DSM-IV-TR). Washington DC: APA Press

American Psychiatric Association (2013). The Diagnostic and Statistical Manual, Fifth Edition (DSM-5). Washington DC: APA Press

Baird, G., \& Norbury, C. F. (2016). Social (pragmatic) communication disorders and autism spectrum disorder. Archives of Disease in Childhood, 101(8), 745-751. https://doi.org/10.1136/archdischild-2014-306944

Bishop, D. V. (1998). Development of the Children's Communication Checklist (CCC): a method for assessing qualitative aspects of communicative impairment in children. Journal of Child Psychology and Psychiatry, and Allied Disciplines, 39(6), 879-891.

Bishop, D. V., \& Baird, G. (2001). Parent and teacher report of pragmatic aspects of communication: use of the children's communication checklist in a clinical setting. Developmental Medicine and Child Neurology, 43(12), 809-818.

Brukner-Wertman, Y., Laor, N., \& Golan, O. (2016). Social (Pragmatic) Communication Disorder and Its Relation to the Autism Spectrum: Dilemmas Arising From the DSM-5 Classification. Journal of Autism and Developmental Disorders. https://doi.org/10.1007/s10803-016-2814-5

Clark, L. A., \& Watson, D. (1995). Constructing validity: Basic issues in objective scale development. Psychological Assessment, 7(3), 309-319. https://doi.org/10.1037/10403590.7.3.309

Constantino, J. N., \& Todd, R. D. (2003). Autistic traits in the general population: a twin study. Archives of General Psychiatry, 60(5), 524-530. https://doi.org/10.1001/archpsyc.60.5.524

Feighner, J. P., Robins, E., Guze, S. B., Woodruff, R. A., Winokur, G., \& Munoz, R. (1972). Diagnostic Criteria for Use in Psychiatric Research. Archives of General Psychiatry, 26(1), 57-63. https://doi.org/10.1001/archpsyc.1972.01750190059011 
Gibson, J., Adams, C., Lockton, E., \& Green, J. (2013). Social communication disorder outside autism? A diagnostic classification approach to delineating pragmatic language impairment, high functioning autism and specific language impairment. Journal of Child Psychology and Psychiatry, and Allied Disciplines, 54(11), 1186-1197. https://doi.org/10.1111/jcpp.12079

Goodman, R. (1997). The Strengths and Difficulties Questionnaire: A Research Note. Journal of Child Psychology and Psychiatry, 38(5), 581-586. https://doi.org/10.1111/j.14697610.1997.tb01545.x

Goodman, R. (2001). Psychometric properties of the strengths and difficulties questionnaire. Journal of the American Academy of Child and Adolescent Psychiatry, 40(11), 1337-1345. https://doi.org/10.1097/00004583-200111000-00015

Happé, F. (2011). Criteria, categories, and continua: autism and related disorders in DSM-5. Journal of the American Academy of Child and Adolescent Psychiatry, 50(6), 540-542. https://doi.org/10.1016/j.jaac.2011.03.015

Happé, F., Ronald, A., \& Plomin, R. (2006). Time to give up on a single explanation for autism. Nature Neuroscience, 9(10), 1218-1220. https://doi.org/10.1038/nn1770

Huerta, M., Bishop, S. L., Duncan, A., Hus, V., \& Lord, C. (2012). Application of DSM-5 criteria for autism spectrum disorder to three samples of children with DSM-IV-TR diagnoses of pervasive developmental disorders. The American Journal of Psychiatry, 169(10), 10561064. https://doi.org/10.1176/appi.ajp.2012.12020276

Kendler, K. S. (1980). The Nosologic Validity of Paranoia (Simple Delusional Disorder): A Review. Archives of General Psychiatry, 37(6), 699-706. https://doi.org/10.1001/archpsyc.1980.01780190097012

Kim, Y. S., Fombonne, E., Koh, Y.-J., Kim, S.-J., Cheon, K.-A., \& Leventhal, B. L. (2014). A comparison of DSM-IV-TR pervasive developmental disorder and DSM-5 autism spectrum 
disorder prevalence in an epidemiologic sample. Journal of the American Academy of Child and Adolescent Psychiatry, 53(5), 500-508. https://doi.org/10.1016/j.jaac.2013.12.021

Lord, C., \& Bishop, S. L. (2015). Recent advances in autism research as reflected in DSM-5 criteria for autism spectrum disorder. Annual Review of Clinical Psychology, 11, 53-70. https://doi.org/10.1146/annurev-clinpsy-032814-112745

Mandy, W., Charman, T., Gilmour, J., \& Skuse, D. (2011). Toward specifying pervasive developmental disorder-not otherwise specified. Autism Research: Official Journal of the International Society for Autism Research, 4(2), 121-131. https://doi.org/10.1002/aur.178

Mandy, W., Chilvers, R., Chowdhury, U., Salter, G., Seigal, A., \& Skuse, D. (2012). Sex differences in autism spectrum disorder: evidence from a large sample of children and adolescents. Journal of Autism and Developmental Disorders, 42(7), 1304-1313. https://doi.org/10.1007/s10803-011-1356-0

Mandy, W. P. L., Charman, T., \& Skuse, D. H. (2012). Testing the construct validity of proposed criteria for DSM-5 autism spectrum disorder. Journal of the American Academy of Child and Adolescent Psychiatry, 51(1), 41-50. https://doi.org/10.1016/j.jaac.2011.10.013

Mandy, W. P. L., \& Skuse, D. H. (2008). Research review: What is the association between the social-communication element of autism and repetitive interests, behaviours and activities? Journal of Child Psychology and Psychiatry, and Allied Disciplines, 49(8), 795-808. https://doi.org/10.1111/j.1469-7610.2008.01911.x

Mandy, W., Skuse, D., Steer, C., St Pourcain, B., \& Oliver, B. R. (2013). Oppositionality and socioemotional competence: interacting risk factors in the development of childhood conduct disorder symptoms. Journal of the American Academy of Child and Adolescent Psychiatry, 52(7), 718-727. https://doi.org/10.1016/j.jaac.2013.04.011

National Institute for Health and Clinical Excellent (2011). Autism spectrum disorder in under 19s: recognition, referral and diagnosis. NICE Guidelines (CG128) 
Norbury, C. F. (2014). Practitioner review: Social (pragmatic) communication disorder conceptualization, evidence and clinical implications. Journal of Child Psychology and Psychiatry, and Allied Disciplines, 55(3), 204-216. https://doi.org/10.1111/jcpp.12154

Pickard, H., Rijsdijk, F., Happé, F., \& Mandy, W. (n.d.). Are Social and Communication Difficulties a Risk Factor for the Development of Social Anxiety? Journal of the American Academy of Child \& Adolescent Psychiatry. https://doi.org/10.1016/j.jaac.2017.01.007

Rutter, M. (1978). Diagnosis and definition of childhood autism. Journal of Autism and Childhood Schizophrenia, 8(2), 139-161.

Simonoff, E., Jones, C. R. G., Baird, G., Pickles, A., Happé, F., \& Charman, T. (2013). The persistence and stability of psychiatric problems in adolescents with autism spectrum disorders. Journal of Child Psychology and Psychiatry, and Allied Disciplines, 54(2), 186194. https://doi.org/10.1111/j.1469-7610.2012.02606.x

Skuse, D., Warrington, R., Bishop, D., Chowdhury, U., Lau, J., Mandy, W., \& Place, M. (2004). The developmental, dimensional and diagnostic interview (3di): a novel computerized assessment for autism spectrum disorders. Journal of the American Academy of Child and Adolescent Psychiatry, 43(5), 548-558. https://doi.org/10.1097/00004583-200405000-00008

St. Pourcain, B., Mandy, W. P., Heron, J., Golding, J., Davey Smith, G., \& Skuse, D. H. (2011). Links Between Co-occurring Social-Communication and Hyperactive-Inattentive Trait Trajectories. Journal of the American Academy of Child \& Adolescent Psychiatry, 50(9), 892-902.e5. https://doi.org/10.1016/j.jaac.2011.05.015

StataCorp. 2015. Stata Statistical Software: Release 14. College Station, TX: StataCorp LP Swineford, L. B., Thurm, A., Baird, G., Wetherby, A. M., \& Swedo, S. (2014). Social (pragmatic) communication disorder: a research review of this new DSM-5 diagnostic category. Journal of Neurodevelopmental Disorders, 6(1), 41. https://doi.org/10.1186/1866-1955-6-41 
Szatmari, P. (2000). The classification of autism, Asperger's syndrome, and pervasive developmental disorder. Canadian Journal of Psychiatry. Revue Canadienne De Psychiatrie, 45(8), 731-738. https://doi.org/10.1177/070674370004500806

Tavakol, M., \& Dennick, R. (2011). Making sense of Cronbach's alpha. International Journal of Medical Education, 2, 53-55. https://doi.org/10.5116/ijme.4dfb.8dfd 
Table 1 - Social (Pragmatic) Communication Disorder Diagnostic Algorithm

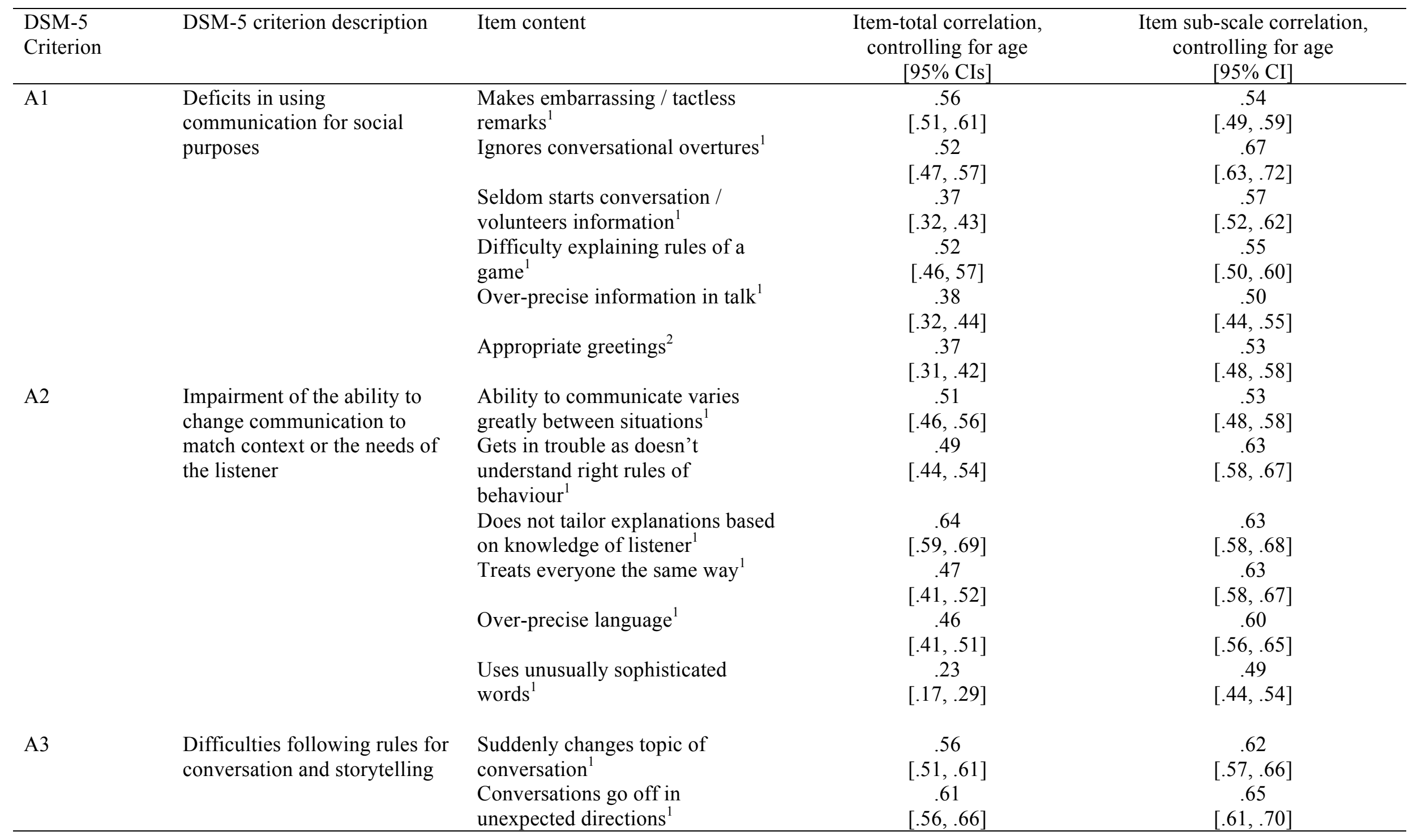




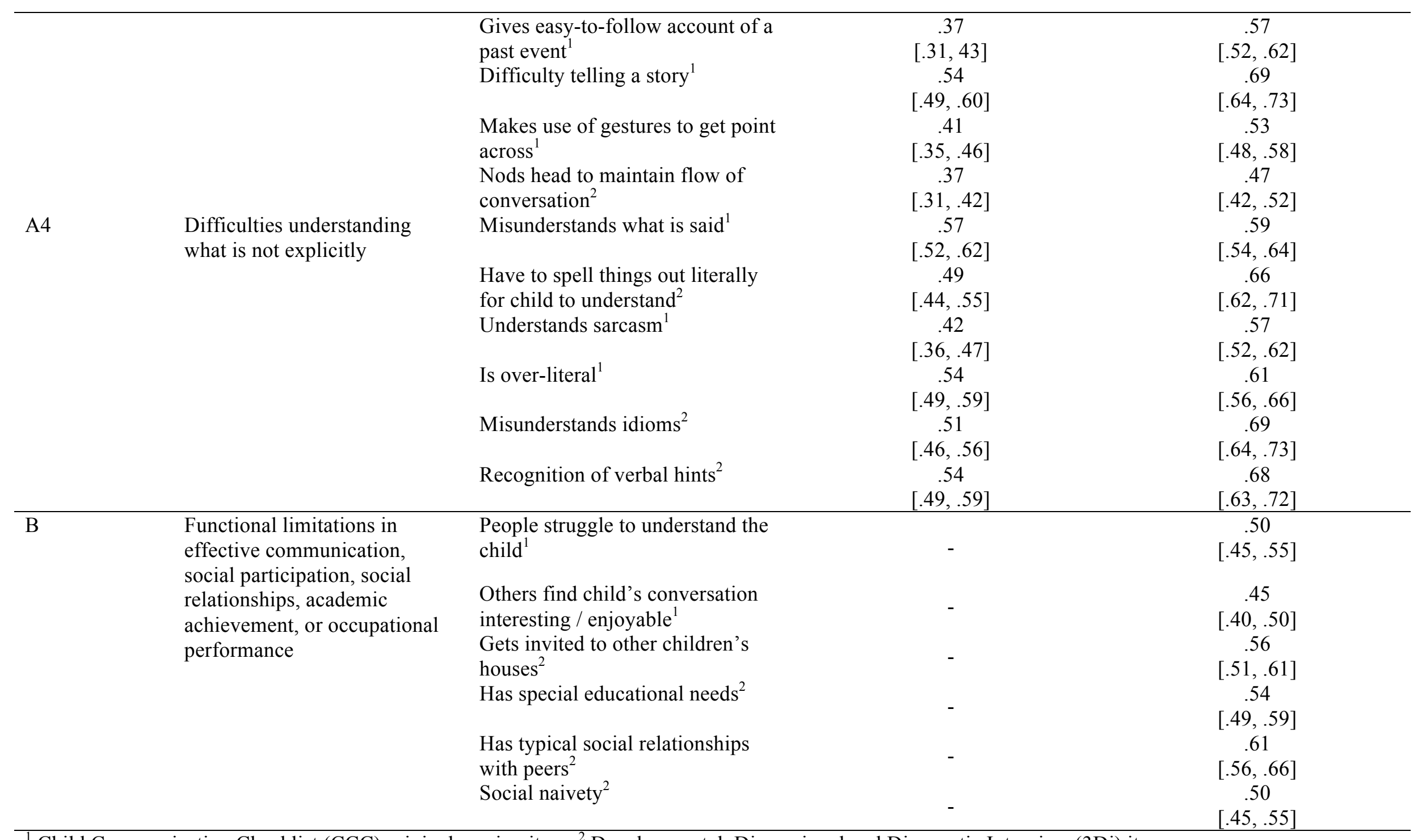

${ }^{1}$ Child Communication Checklist (CCC) original version item; ${ }^{2}$ Developmental, Dimensional and Diagnostic Interview (3Di) item. 
Table 2 - Agreement between DSM-5 and DSM-IV-TR classifications

DSM-IV-TR

No PDD PDD-NOS $\begin{gathered}\text { Asperger's } \\ \text { Disorder }\end{gathered} \quad$ Autistic disorder Total

\begin{tabular}{|c|c|c|c|c|c|c|}
\hline & $\begin{array}{l}\text { No ASD or } \\
\text { SPCD } \\
(\text { Row \%) }\end{array}$ & $\begin{array}{c}144 \\
(75.0 \%)\end{array}$ & $\begin{array}{c}37 \\
(19.3 \%)\end{array}$ & $\begin{array}{c}7 \\
(3.7 \%)\end{array}$ & $\begin{array}{c}2 \\
(2.1 \%)\end{array}$ & $\begin{array}{c}192 \\
(100 \%)\end{array}$ \\
\hline \multirow[t]{3}{*}{ DSM-5 } & $\begin{array}{l}\text { SPCD } \\
(\text { Row \%) }\end{array}$ & $\begin{array}{c}38 \\
(43.2 \%)\end{array}$ & $\begin{array}{c}26 \\
(29.6 \%)\end{array}$ & $\begin{array}{c}10 \\
(11.4 \%)\end{array}$ & $\begin{array}{c}14 \\
(15.9 \%)\end{array}$ & $\begin{array}{c}88 \\
(100 \%)\end{array}$ \\
\hline & $\begin{array}{l}\text { ASD } \\
(\text { Row \%) }\end{array}$ & $\begin{array}{c}84 \\
(10.5 \%)\end{array}$ & $\begin{array}{c}156 \\
(19.5 \%)\end{array}$ & $\begin{array}{c}272 \\
(34.0 \%)\end{array}$ & $\begin{array}{c}289 \\
(36.1 \%)\end{array}$ & $\begin{array}{c}801 \\
(100 \%)\end{array}$ \\
\hline & & 266 & 219 & 289 & 307 & \\
\hline
\end{tabular}

DSM-IV-TR = Diagnostic and Statistical Manual, Fourth Edition Text Revision; DSM-5 = Diagnostic and Statistical Manual, Fifth Edition; PDD = Pervasive Developmental Disorder; PDD-NOS = Pervasive developmental disorder - not otherwise specified; ASD = autism spectrum disorder; SPCD = Social (Pragmatic) Communication Disorder 
Table 3 - Demographic, language and autistic characteristics of sample by group

\begin{tabular}{|c|c|c|c|c|c|}
\hline & No ASD or SPCD & SPCD & ASD & $\begin{array}{l}\text { Significance of } \\
\text { group comparison }\end{array}$ & $\begin{array}{c}\text { Post hoc } \\
\text { comparisons }\end{array}$ \\
\hline $\begin{array}{l}\text { Mean age }(\mathrm{SD}) \text { in } \\
\text { years }\end{array}$ & $\begin{array}{l}10.0 \\
(3.5)\end{array}$ & $\begin{array}{l}9.7 \\
(3.5)\end{array}$ & $\begin{array}{l}10.3 \\
(3.3)\end{array}$ & $\mathrm{P}=.243$ & \\
\hline Proportion male & $77.6 \%$ & $83.0 \%$ & $82.0 \%$ & $\mathrm{P}=.340$ & \\
\hline CCC Speech & $\begin{array}{l}32.9 \\
(3.6)\end{array}$ & $\begin{array}{l}29.9 \\
(5.2)\end{array}$ & $\begin{array}{l}30.9 \\
(4.5)\end{array}$ & $\mathrm{P}<.001$ & $\begin{array}{c}\mathrm{SPCD}=\mathrm{ASD}<\text { no- } \\
\mathrm{ASD}\end{array}$ \\
\hline CCC Syntax & $\begin{array}{l}31.0 \\
(1.4)\end{array}$ & $\begin{array}{l}29.9 \\
(2.5)\end{array}$ & $\begin{array}{l}30.3 \\
(2.1)\end{array}$ & $\mathrm{P}<.001$ & $\begin{array}{c}\mathrm{SPCD}=\mathrm{ASD}<\text { no- } \\
\mathrm{ASD}\end{array}$ \\
\hline $\begin{array}{l}\text { CCC Social } \\
\text { Relationships }\end{array}$ & $\begin{array}{l}28.8 \\
(3.7)\end{array}$ & $\begin{array}{l}25.7 \\
(4.6)\end{array}$ & $\begin{array}{l}23.7 \\
(4.0)\end{array}$ & $\mathrm{P}<.001$ & $\begin{array}{c}\mathrm{ASD}<\mathrm{SPCD}<\text { no- } \\
\mathrm{ASD}\end{array}$ \\
\hline CCC Interests & $\begin{array}{l}30.1 \\
(2.5)\end{array}$ & $\begin{array}{l}29.3 \\
(2.8)\end{array}$ & $\begin{array}{l}27.2 \\
(2.7)\end{array}$ & $\mathrm{P}<.001$ & $\begin{array}{c}\mathrm{ASD}<\mathrm{SPCD}=\text { no- } \\
\mathrm{ASD}\end{array}$ \\
\hline
\end{tabular}

$\mathrm{ASD}=$ autism spectrum disorder; $\mathrm{CCC}=$ Child Communication Checklist; SPCD $=$ Social (Pragmatic) Communication Disorder 
Table 4 - Proportion scoring in the abnormal range on the Strengths and Difficulties Questionnaire by group

\begin{tabular}{|c|c|c|c|c|c|c|}
\hline & $\begin{array}{c}\text { No ASD or } \\
\text { SPCD } \\
n=107\end{array}$ & $\begin{array}{l}\text { SPCD } \\
n=52\end{array}$ & $\begin{array}{l}\text { ASD } \\
\mathrm{n}=453\end{array}$ & & $\begin{array}{l}\text { SPCD v non- } \\
\text { ASD }\end{array}$ & SPCD v ASD \\
\hline Emotional Problems & $\begin{array}{c}38 \\
(35.2 \%)\end{array}$ & $\begin{array}{c}23 \\
(44.3 \%)\end{array}$ & $\begin{array}{c}266 \\
(58.7 \%)\end{array}$ & $\mathrm{P}<.001$ & $\begin{array}{c}1.5 \\
{[.7,3.0]}\end{array}$ & $\begin{array}{c}.6 \\
{[.3,1.0]}\end{array}$ \\
\hline Hyperactivity & $\begin{array}{c}33 \\
(30.8 \%)\end{array}$ & $\begin{array}{c}31 \\
(59.6 \%)\end{array}$ & $\begin{array}{c}278 \\
(61.4 \%)\end{array}$ & $\mathrm{P}<.001$ & $\begin{array}{c}3.3 \\
{[1.6,7.0]}\end{array}$ & $\begin{array}{c}.9 \\
{[.5,1.8]}\end{array}$ \\
\hline
\end{tabular}




\begin{tabular}{|c|c|c|c|c|c|c|}
\hline Peer Problems & $\begin{array}{c}50 \\
(46.7 \%)\end{array}$ & $\begin{array}{c}28 \\
(53.9 \%)\end{array}$ & $\begin{array}{c}346 \\
(76.4 \%)\end{array}$ & $\mathrm{P}<.001$ & $\begin{array}{c}1.3 \\
{[.65,2.7]}\end{array}$ & $\begin{array}{c}.4 \\
{[.2, .7]}\end{array}$ \\
\hline
\end{tabular}

$\mathrm{ASD}=$ autism spectrum disorder; $\mathrm{CCC}=$ Child Communication Checklist; SPCD = Social (Pragmatic) Communication Disorder 
Figure 1 - Autistic social and non-social traits by group

Note: Scores are standardised as z-scores using the sample mean and standard deviation, with lower scores denoting greater impairment

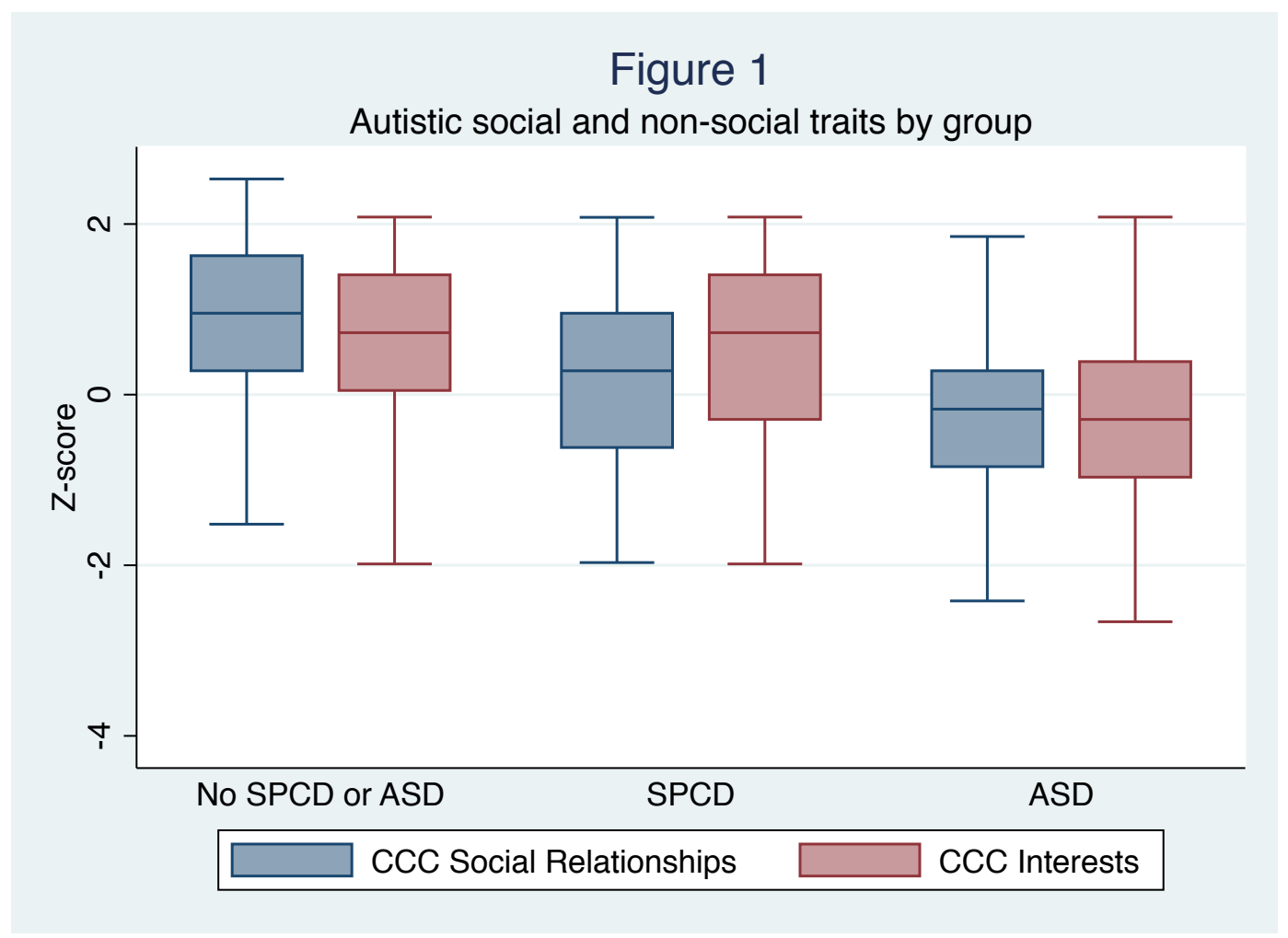


Key points

- Social (pragmatic) communication disorder (SPCD) is a new DSM-5 diagnosis characterised by difficulties with the social aspects of verbal and non-verbal communication.

- Because it was only recently introduced, it is not known whether SPCD is a valid diagnosis. Therefore we identified and described cases of SPCD in relation to ASD and clinical controls, to increase understanding of SPCD's validity.

- We did not find evidence that SPCD is qualitatively distinct from ASD. Rather, it appears to exist on the borderlands of the autism spectrum, applying to people who have autistic traits that are just below the threshold for an ASD.

- SPCD may have utility as a diagnostic home for people with 'nearly autism' - those who narrowly miss meeting criteria for ASD, but who nevertheless have substantial clinical needs. 